\title{
Solvability of boundary value problem with $p$-Laplacian at resonance
}

\section{Weihua Jiang*}

\section{"Correspondence:}

weihuajiang@hebust.edu.cn

College of Sciences, Hebei

University of Science and

Technology, Shijiazhuang, Hebei

050018, P.R. China

\begin{abstract}
By generalizing the extension of the continuous theorem of $\mathrm{Ge}$ and Ren and constructing suitable Banach spaces and operators, we investigate the existence of solutions for $p$-Laplacian boundary value problems at resonance. An example is given to illustrate our results.

MSC: 34B15

Keywords: continuous theorem; resonance; $p$-Laplacian; boundary value problem
\end{abstract}

\section{Introduction}

In this paper, we will study the boundary value problem

$$
\left\{\begin{array}{l}
\left(\varphi_{p}\left(u^{\prime \prime}\right)\right)^{\prime}(t)=f\left(t, u(t), u^{\prime}(t), u^{\prime \prime}(t)\right) \\
u(0)=u^{\prime \prime}(0)=0, \quad u^{\prime}(1)=\int_{0}^{1} k(t) u^{\prime}(t) d t
\end{array}\right.
$$

and

$$
\left\{\begin{array}{l}
\left(\varphi_{p}\left(u^{\prime \prime}\right)\right)^{\prime}(t)=f\left(t, u(t), u^{\prime}(t), u^{\prime \prime}(t)\right), \\
u^{\prime \prime}(0)=0, \quad u^{\prime}(0)=\int_{0}^{1} g(t) u^{\prime}(t) d t, \quad u^{\prime}(1)=\int_{0}^{1} h(t) u^{\prime}(t) d t,
\end{array}\right.
$$

where $\varphi_{p}(s)=|s|^{p-2} s, p>1, \int_{0}^{1} k(t) d t=1, \int_{0}^{1} g(t) d t=1, \int_{0}^{1} h(t) d t=1$.

A boundary value problem is said to be a resonance one if the corresponding homogeneous boundary value problem has a non-trivial solution. Mawhin's continuous theorem [1] is an effective tool to solve this kind of problems when the differential operator is linear, see [2-10] and references cited therein. But it does not work for nonlinear cases such as boundary value problems with a $p$-Laplacian, which attracted the attention of mathematicians in recent years [11-15]. Ge and Ren extended Mawhin's continuous theorem [15] and many authors used their results to solve boundary value problems with a $p$-Laplacian, see $[16,17]$. In this new theorem, two projectors $P$ and $Q$ must be constructed. But it is difficult to give the projector $Q$ in many boundary value problems with a $p$-Laplacian. In this paper, we generalize the extension of the continuous theorem and show that the $p$-Laplacian problem is solvable when $Q$ is not a projector. And we will use this new theorem to discuss problems (1.1) and (1.2), respectively.

In this paper, we will always suppose that

O2014 Jiang; licensee Springer. This is an Open Access article distributed under the terms of the Creative Commons Attribution License (http://creativecommons.org/licenses/by/2.0), which permits unrestricted use, distribution, and reproduction in any medium, provided the original work is properly cited. 
$\left(\mathrm{H}_{1}\right) \quad k(t), g(t), h(t) \in L^{1}[0,1]$ are nonnegative and $\|k\|_{1}=\|g\|_{1}=\|h\|_{1}=1$, where $\|k\|_{1}:=$ $\int_{0}^{1}|k(t)| d t$.

$\left(\mathrm{H}_{2}\right) f(t, u, v, w)$ is continuous in $[0,1] \times \mathbb{R}^{3}$.

\section{Preliminaries}

Definition 2.1 [15] Let $X$ and $Y$ be two Banach spaces with norms $\|\cdot\|_{X},\|\cdot\|_{Y}$, respectively. A continuous operator $M: X \cap \operatorname{dom} M \rightarrow Y$ is said to be quasi-linear if

(i) $\operatorname{Im} M:=M(X \cap \operatorname{dom} M)$ is a closed subset of $Y$,

(ii) $\operatorname{Ker} M:=\{x \in X \cap \operatorname{dom} M: M x=0\}$ is linearly homeomorphic to $\mathbb{R}^{n}, n<\infty$, where $\operatorname{dom} M$ denote the domain of the operator $M$.

Let $X_{1}=\operatorname{Ker} M$ and $X_{2}$ be the complement space of $X_{1}$ in $X$, then $X=X_{1} \oplus X_{2}$. Let $P$ : $X \rightarrow X_{1}$ be a projector and $\Omega \subset X$ an open and bounded set with the origin $\theta \in \Omega$.

Definition 2.2 Suppose $N_{\lambda}: \bar{\Omega} \rightarrow Y, \lambda \in[0,1]$ is a continuous and bounded operator. Denote $N_{1}$ by $N$. Let $\Sigma_{\lambda}=\left\{x \in \bar{\Omega} \cap \operatorname{dom} M: M x=N_{\lambda} x\right\}$. $N_{\lambda}$ is said to be $M$-quasi-compact in $\bar{\Omega}$ if there exists a vector subspace $Y_{1}$ of $Y$ satisfying $\operatorname{dim} Y_{1}=\operatorname{dim} X_{1}$ and two operators $Q, R$ with $Q: Y \rightarrow Y_{1}, Q Y=Y_{1}$, being continuous, bounded, and satisfying $Q(I-Q)=0$, $R: \bar{\Omega} \times[0,1] \rightarrow X_{2} \cap \operatorname{dom} M$ continuous and compact such that for $\lambda \in[0,1]$,

(a) $(I-Q) N_{\lambda}(\bar{\Omega}) \subset \operatorname{Im} M \subset(I-Q) Y$,

(b) $Q N_{\lambda} x=\theta, \lambda \in(0,1) \Leftrightarrow Q N x=\theta$,

(c) $R(\cdot, 0)$ is the zero operator and $\left.R(\cdot, \lambda)\right|_{\Sigma_{\lambda}}=\left.(I-P)\right|_{\Sigma_{\lambda}}$,

(d) $M[P+R(\cdot, \lambda)]=(I-Q) N_{\lambda}$.

Theorem 2.1 Let $X$ and $Y$ be two Banach spaces with the norms $\|\cdot\|_{X},\|\cdot\|_{Y}$, respectively, and let $\Omega \subset X$ be an open and bounded nonempty set. Suppose

$$
M: X \cap \operatorname{dom} M \rightarrow Y
$$

is a quasi-linear operator and that $N_{\lambda}: \bar{\Omega} \rightarrow Y, \lambda \in[0,1]$ is M-quasi-compact. In addition, if the following conditions hold:

$\left(\mathrm{C}_{1}\right) \quad M x \neq N_{\lambda} x, \forall x \in \partial \Omega \cap \operatorname{dom} M, \lambda \in(0,1)$,

$\left(C_{2}\right) \operatorname{deg}\{J Q N, \Omega \cap \operatorname{Ker} M, 0\} \neq 0$,

then the abstract equation $M x=N x$ has at least one solution in $\operatorname{dom} M \cap \bar{\Omega}$, where $N=N_{1}$, $J: \operatorname{Im} Q \rightarrow \operatorname{Ker} M$ is a homeomorphism with $J(\theta)=\theta$.

Proof The proof is similar to the one of Lemma 2.1 and Theorem 2.1 in [15].

We can easily get the following inequalities.

Lemma 2.1 For any $u, v \geq 0$, we have

(1) $\varphi_{p}(u+v) \leq \varphi_{p}(u)+\varphi_{p}(v), 1<p \leq 2$.

(2) $\varphi_{p}(u+v) \leq 2^{p-2}\left(\varphi_{p}(u)+\varphi_{p}(v)\right), p \geq 2$.

In the following, we will always suppose that $q$ satisfies $1 / p+1 / q=1$. 


\section{The existence of a solution for problem (1.1)}

Let $X=C^{2}[0,1]$ with norm $\|u\|=\max \left\{\|u\|_{\infty},\left\|u^{\prime}\right\|_{\infty},\left\|u^{\prime \prime}\right\|_{\infty}\right\}, Y=C[0,1] \times C[0,1]$ with norm $\left\|\left(y_{1}, y_{2}\right)\right\|=\max \left\{\left\|y_{1}\right\|_{\infty},\left\|y_{2}\right\|_{\infty}\right\}$, where $\|y\|_{\infty}=\max _{t \in[0,1]}|y(t)|$. We know that $(X,\|\cdot\|)$ and $(Y,\|\cdot\|)$ are Banach spaces.

Define operators $M: X \cap \operatorname{dom} M \rightarrow Y, N_{\lambda}: X \rightarrow Y$ as follows:

$$
M u=\left[\begin{array}{c}
\left(\varphi_{p}\left(u^{\prime \prime}\right)\right)^{\prime}(t) \\
T\left(\varphi_{p}\left(u^{\prime \prime}\right)\right)^{\prime}(t)
\end{array}\right], \quad N_{\lambda} u=\left[\begin{array}{c}
\lambda f\left(t, u(t), u^{\prime}(t), u^{\prime \prime}(t)\right) \\
0
\end{array}\right]
$$

where $T y=c, y \in C[0,1], c$ satisfying

$$
\begin{aligned}
& \int_{0}^{1} k(t) \int_{t}^{1} \varphi_{q}\left(\int_{0}^{s} y(r)-c d r\right) d s d t=0, \\
& \operatorname{dom} M=\left\{u \in X \mid \varphi_{p}\left(u^{\prime \prime}\right) \in C^{1}[0,1], u(0)=u^{\prime \prime}(0)=0\right\} .
\end{aligned}
$$

Lemma 3.1 For $y \in C[0,1]$, there is only one constant $c \in \mathbb{R}$ such that $T y=c$ with $|c| \leq\|y\|_{\infty}$ and that $T: C[0,1] \rightarrow \mathbb{R}$ is continuous.

Proof For $y \in C[0,1]$, let

$$
F(c)=\int_{0}^{1} k(t) \int_{t}^{1} \varphi_{q}\left(\int_{0}^{s}(y(r)-c) d r\right) d s d t
$$

Obviously, $F(c)$ is continuous and strictly decreasing in $\mathbb{R}$. Take $a=\min _{t \in[0,1]} y(t), b=$ $\max _{t \in[0,1]} y(t)$. It is easy to see that $F(a) \geq 0, F(b) \leq 0$. Thus, there exists a unique constant $c \in[a, b]$ such that $F(c)=0$, i.e. there is only one constant $c \in \mathbb{R}$ such that $T y=c$ with $|c| \leq\|y\|_{\infty}$.

For $y_{1}, y_{2} \in C[0,1]$, assume $T y_{1}=c_{1}, T y_{2}=c_{2}$. By $k(t) \geq 0, \int_{0}^{1} k(t) d t=1$ and $\varphi_{q}$ being strictly increasing, we obtain, if $c_{2}-c_{1}>\max _{t \in[0,1]}\left(y_{2}(t)-y_{1}(t)\right)$, then

$$
\begin{aligned}
0 & =\int_{0}^{1} k(t) \int_{t}^{1} \varphi_{q}\left(\int_{0}^{s}\left(y_{2}(r)-c_{2}\right) d r\right) d s d t \\
& =\int_{0}^{1} k(t) \int_{t}^{1} \varphi_{q}\left(\int_{0}^{s}\left[\left(y_{1}(r)-c_{1}\right)+\left(y_{2}(r)-y_{1}(r)-\left(c_{2}-c_{1}\right)\right) d r\right]\right) d s d t \\
& <\int_{0}^{1} k(t) \int_{t}^{1} \varphi_{q}\left(\int_{0}^{s}\left(y_{1}(r)-c_{1}\right) d r\right) d s d t=0 .
\end{aligned}
$$

This is a contradiction. On the other hand, if $c_{2}-c_{1}<\min _{t \in[0,1]}\left(y_{2}(t)-y_{1}(t)\right)$, then

$$
\begin{aligned}
0 & =\int_{0}^{1} k(t) \int_{t}^{1} \varphi_{q}\left(\int_{0}^{s}\left(y_{2}(r)-c_{2}\right) d r\right) d s d t \\
& =\int_{0}^{1} k(t) \int_{t}^{1} \varphi_{q}\left(\int_{0}^{s}\left[\left(y_{1}(r)-c_{1}\right)+\left(y_{2}(r)-y_{1}(r)-\left(c_{2}-c_{1}\right)\right) d r\right]\right) d s d t \\
& >\int_{0}^{1} k(t) \int_{t}^{1} \varphi_{q}\left(\int_{0}^{s}\left(y_{1}(r)-c_{1}\right) d r\right) d s d t=0 .
\end{aligned}
$$


This is a contradiction, too. So, we have $\min _{t \in[0,1]}\left(y_{2}(t)-y_{1}(t)\right) \leq c_{2}-c_{1} \leq \max _{t \in[0,1]}\left(y_{2}(t)-\right.$ $\left.y_{1}(t)\right)$, i.e. $\left|c_{2}-c_{1}\right| \leq\left\|y_{2}-y_{1}\right\|_{\infty}$. So, $T: C[0,1] \rightarrow \mathbb{R}$ is continuous. The proof is completed.

It is clear that $u \in \operatorname{dom} M$ is a solution if and only if it satisfies $M u=N u$, where $N=N_{1}$. For convenience, let $(a, b)^{L}:=\left[\begin{array}{l}a \\ b\end{array}\right]$.

Lemma 3.2 $M$ is a quasi-linear operator.

Proof It is easy to see that $\operatorname{Ker} M=\{b t \mid b \in \mathbb{R}\}:=X_{1}$.

For $u \in X \cap \operatorname{dom} M$, if $M u=(y, c)^{L}$, then $c$ satisfies (3.1). On the other hand, if $y \in C[0,1]$, $T y=c$, take

$$
u(t)=\int_{0}^{t}(t-s) \varphi_{q}\left(\int_{0}^{s} y(r) d r\right) d s
$$

By a simple calculation, we get $u \in X \cap \operatorname{dom} M$ and $M u=(y, c)^{L}$. Thus

$$
\operatorname{Im} M=\left\{(y, c)^{L} \mid y \in C[0,1], c \text { satisfies (3.1) }\right\} .
$$

By the continuity of $T$, we find that $\operatorname{Im} M \subset Y$ is closed. So, $M$ is quasi-linear. The proof is completed.

Lemma 3.3 $T(c)=c, T(y+c)=T(y)+c, T(c y)=c T(y), c \in \mathbb{R}, y \in C[0,1]$.

Proof The proof is simple. Therefore, we omit it.

Take a projector $P: X \rightarrow X_{1}$ and an operator $Q: Y \rightarrow Y_{1}$ as follows:

$$
(P u)(t)=u^{\prime}(0) t, \quad Q\left(y, y_{1}\right)^{L}=\left(0, T y_{1}-T y\right)^{L},
$$

where $Y_{1}=\left\{(0, c)^{L} \mid c \in \mathbb{R}\right\}$. Obviously, $Q Y=Y_{1}$, and $\operatorname{dim} Y_{1}=\operatorname{dim} X_{1}$.

By the continuity and boundedness of $T$, we can easily see that $Q$ is continuous and bounded in $Y$. It follows from Lemma 3.3 that $Q(I-Q)\left(y, y_{1}\right)^{L}=(0,0)^{L}, y, y_{1} \in C[0,1]$.

Define an operator $R: X \times[0,1] \rightarrow X_{2}$ as

$$
R(u, \lambda)(t)=\int_{0}^{t}(t-s) \varphi_{q}\left(\int_{0}^{s} \lambda f\left(r, u(r), u^{\prime}(r), u^{\prime \prime}(r)\right) d r\right) d s,
$$

where $\operatorname{Ker} M \oplus X_{2}=X$. By $\left(\mathrm{H}_{2}\right)$ and the Arzela-Asscoli theorem, we can easily see that $R: \bar{\Omega} \times[0,1] \rightarrow X_{2} \cap \operatorname{dom} M$ is continuous and compact, where $\Omega \subset X$ is an open bounded set.

Lemma 3.4 Assume that $\Omega \subset X$ is an open bounded set. Then $N_{\lambda}$ is M-quasi-compact in $\bar{\Omega}$. 
Proof It is clear that $\operatorname{Im} P=\operatorname{Ker} M, Q N_{\lambda} x=\theta, \lambda \in(0,1) \Leftrightarrow Q N x=\theta$ and $R(\cdot, 0)=0$. For $u \in \bar{\Omega}$,

$$
\begin{aligned}
(I-Q) N_{\lambda} u & =\left[\begin{array}{c}
\lambda f\left(t, u(t), u^{\prime}(t), u^{\prime \prime}(t)\right) \\
0
\end{array}\right]-\left[\begin{array}{c}
0 \\
-T\left[\lambda f\left(t, u(t), u^{\prime}(t), u^{\prime \prime}(t)\right)\right]
\end{array}\right] \\
& =\left[\begin{array}{c}
\lambda f\left(t, u(t), u^{\prime}(t), u^{\prime \prime}(t)\right) \\
T\left[\lambda f\left(t, u(t), u^{\prime}(t), u^{\prime \prime}(t)\right)\right]
\end{array}\right] \in \operatorname{Im} M .
\end{aligned}
$$

Since $\operatorname{Im} M \subset \operatorname{Ker} Q$ and $y=Q y+(I-Q) y$, we obtain $\operatorname{Im} M \subset(I-Q) Y$. Thus, $(I-Q) N_{\lambda}(\bar{\Omega}) \subset$ $\operatorname{Im} M \subset(I-Q) Y$.

For $u \in \Sigma_{\lambda}=\left\{u \in \bar{\Omega} \cap \operatorname{dom} M: M u=N_{\lambda} u\right\}$, we get

$$
\begin{aligned}
R(u, \lambda) & =\int_{0}^{t}(t-s) \varphi_{q}\left(\int_{0}^{s} \lambda f\left(r, u(r), u^{\prime}(r), u^{\prime \prime}(r)\right) d r\right) d s \\
& =\int_{0}^{t}(t-s) \varphi_{q}\left(\int_{0}^{s}\left(\varphi_{p}\left(u^{\prime \prime}\right)\right)^{\prime}\right) d s \\
& =u(t)-u^{\prime}(0) t=(I-P) u,
\end{aligned}
$$

i.e. Definition 2.2(c) holds. For $u \in \bar{\Omega}$, we have

$$
M[P u+R(u, \lambda)]=\left[\begin{array}{c}
\lambda f\left(t, u(t), u^{\prime}(t), u^{\prime \prime}(t)\right) \\
T\left[\lambda f\left(t, u(t), u^{\prime}(t), u^{\prime \prime}(t)\right)\right]
\end{array}\right]=(I-Q) N_{\lambda} u .
$$

So, Definition 2.2(d) holds. Therefore, $N_{\lambda}$ is $M$-quasi-compact in $\bar{\Omega}$. The proof is completed.

Theorem 3.1 Assume that the following conditions hold.

$\left(\mathrm{H}_{3}\right)$ There exists a nonnegative constant $K$ such that one of (1) and (2) holds:

(1) $B f(t, A, B, C)>0, t \in[0,1],|B|>K, A, C \in \mathbb{R}$,

(2) $B f(t, A, B, C)<0, t \in[0,1],|B|>K, A, C \in \mathbb{R}$.

$\left(\mathrm{H}_{4}\right)$ There exist nonnegative functions $a(t), b(t), c(t), e(t) \in L^{1}[0,1]$ such that

$$
|f(t, x, y, z)| \leq a(t) \varphi_{p}(|x|)+b(t) \varphi_{p}(|y|)+c(t) \varphi_{p}(|z|)+e(t), \quad t \in[0,1], x, y, z \in \mathbb{R},
$$

where $\varphi_{q}\left(\|a\|_{1}+\|b\|_{1}+\|c\|_{1}\right)<2^{2-q}$, if $1<p \leq 2 ; \varphi_{q}\left(2^{p-2}\|a\|_{1}+2^{p-2}\|b\|_{1}+\|c\|_{1}\right)<1$, if $p \geq 2$.

Then boundary value problem (1.1) has at least one solution.

In order to prove Theorem 3.1, we show two lemmas.

Lemma 3.5 Suppose $\left(\mathrm{H}_{3}\right)$ and $\left(\mathrm{H}_{4}\right)$ hold. Then the set

$$
\Omega_{1}=\left\{u \in \operatorname{dom} M \mid M u=N_{\lambda} u, \lambda \in(0,1)\right\}
$$

is bounded in X. 
Proof For $u \in \Omega_{1}$, we have $Q N_{\lambda} u=0$, i.e. $T f\left(t, u(t), u^{\prime}(t), u^{\prime \prime}(t)\right)=0$. By $\left(\mathrm{H}_{3}\right)$, there exists a constant $t_{0} \in[0,1]$ such that $\left|u^{\prime}\left(t_{0}\right)\right| \leq K$. Since $u(t)=\int_{0}^{t} u^{\prime}(s) d s, u^{\prime}(t)=u^{\prime}\left(t_{0}\right)+\int_{t_{0}}^{t} u^{\prime \prime}(s) d s$, we have

$$
|u(t)| \leq\left\|u^{\prime}\right\|_{\infty}, \quad\left|u^{\prime}(t)\right| \leq K+\left\|u^{\prime \prime}\right\|_{\infty}, \quad t \in[0,1] .
$$

It follows from $M u=N_{\lambda} u,\left(\mathrm{H}_{4}\right)$, and (3.2) that

$$
\begin{aligned}
\left|u^{\prime \prime}(t)\right| & =\left|\varphi_{q}\left(\int_{0}^{t} \lambda f\left(s, u(s), u^{\prime}(s), u^{\prime \prime}(s)\right) d s\right)\right| \\
& \leq \varphi_{q}\left(\int_{0}^{1} a(t) \varphi_{p}(|u|)+b(t) \varphi_{p}\left(\left|u^{\prime}\right|\right)+c(t) \varphi_{p}\left(\left|u^{\prime \prime}\right|\right)+e(t) d t\right) \\
& \leq \varphi_{q}\left[\left(\|a\|_{1}+\|b\|_{1}\right) \varphi_{p}\left(K+\left\|u^{\prime \prime}\right\|_{\infty}\right)+\|c\|_{1} \varphi_{p}\left(\left\|u^{\prime \prime}\right\|_{\infty}\right)+\|e\|_{1}\right] .
\end{aligned}
$$

If $1<p \leq 2$, by Lemma 2.1, we get

$$
\left|u^{\prime \prime}(t)\right| \leq \varphi_{q}\left(B_{1}+A_{1} \varphi_{p}\left(\left\|u^{\prime \prime}\right\|_{\infty}\right)\right) \leq 2^{q-2}\left[\varphi_{q}\left(B_{1}\right)+\varphi_{q}\left(A_{1}\right)\left\|u^{\prime \prime}\right\|_{\infty}\right],
$$

thus

$$
\left\|u^{\prime \prime}\right\|_{\infty} \leq \frac{2^{q-2} \varphi_{q}\left(B_{1}\right)}{1-2^{q-2} \varphi_{q}\left(A_{1}\right)}
$$

where $B_{1}=\left(\|a\|_{1}+\|b\|_{1}\right) \varphi_{p}(K)+\|e\|_{1}, A_{1}=\|a\|_{1}+\|b\|_{1}+\|c\|_{1}$.

If $p>2$, by Lemma 2.1, we get

$$
\left|u^{\prime \prime}(t)\right| \leq \varphi_{q}\left(B_{2}+A_{2} \varphi_{p}\left(\left\|u^{\prime \prime}\right\|_{\infty}\right)\right) \leq\left[\varphi_{q}\left(B_{2}\right)+\varphi_{q}\left(A_{2}\right)\left\|u^{\prime \prime}\right\|_{\infty}\right]
$$

thus

$$
\left\|u^{\prime \prime}\right\|_{\infty} \leq \frac{\varphi_{q}\left(B_{2}\right)}{1-\varphi_{q}\left(A_{2}\right)}
$$

where $B_{2}=2^{p-2}\left(\|a\|_{1}+\|b\|_{1}\right) \varphi_{p}(K)+\|e\|_{1}, A_{2}=2^{p-2}\left(\|a\|_{1}+\|b\|_{1}\right)+\|c\|_{1}$.

These, together with (3.2), mean that $\Omega_{1}$ is bounded in $X$.

Lemma 3.6 Assume $\left(\mathrm{H}_{3}\right)$ holds. Then

$$
\Omega_{2}=\{u \in \operatorname{Ker} M \mid Q N u=0\}
$$

is bounded in $X$, where $N=N_{1}$.

Proof For $u \in \Omega_{2}$, we have $u=b t$ and $T f(t, b t, b, 0)=0$. By $\left(\mathrm{H}_{3}\right)$, we get $|b| \leq K$. So, $\Omega_{2}$ is bounded. The proof is completed.

Proof of Theorem 3.1 Let $\Omega=\{u \in X \mid\|u\|<r\}$, where $r$ is large enough such that $K<r<$ $+\infty$ and $\Omega \supset \overline{\Omega_{1}}$. 
By Lemmas 3.5 and 3.6, we know $M u \neq N_{\lambda} u, u \in \operatorname{dom} M \cap \partial \Omega$ and $Q N u \neq 0, u \in \operatorname{Ker} M \cap$ $\partial \Omega$.

Let $H(u, \delta)=\rho \delta u+(1-\delta) J Q N u, \delta \in[0,1], u \in \operatorname{Ker} M \cap \bar{\Omega}$, where $J: \operatorname{Im} Q \rightarrow \operatorname{Ker} M$ is a homeomorphism with $J(0, b)^{L}=b t, \rho= \begin{cases}-1, & \text { if }\left(\mathrm{H}_{3}\right)(1) \text { holds, } \\ 1, & \text { if }\left(\mathrm{H}_{3}\right)(2) \text { holds. }\end{cases}$

Define a function $\operatorname{Sgn}(x)= \begin{cases}1, & \text { if } x>0, \\ -1, & \text { if } x<0 .\end{cases}$

For $u \in \operatorname{Ker} M \cap \partial \Omega$, we have $u=b t \neq 0$. Thus

$$
H(u, \delta)=\rho \delta b t+(1-\delta)(-T f(t, b t, b, 0)) t .
$$

If $\delta=1, H(u, 1)=\rho b t \neq 0$. If $\delta=0$, by $Q N u \neq 0$, we get $H(u, 0)=J Q N(b t) \neq 0$. For $0<\delta<1$, we now prove that $H(u, \delta) \neq 0$. Otherwise, if $H(u, \delta)=0$, then

$$
T f(t, b t, b, 0)=\frac{\rho \delta}{1-\delta} b
$$

Since $\|u\|=r>K$, we have $|b|>K$. Thus, $T[b f(t, b t, b, 0)]=b T f(t, b t, b, 0)=\frac{\rho \delta}{1-\delta} b^{2}$. So, we have $\operatorname{Sgn}(b f(t, b t, b, 0))=\operatorname{Sgn}\{T[b f(t, b t, b, 0)]\}=\operatorname{Sgn}\left(\frac{\rho \delta}{1-\delta} b^{2}\right)=\operatorname{Sgn}(\rho)$. A contradiction with the definition of $\rho$. So, $H(u, \delta) \neq 0, u \in \operatorname{Ker} M \cap \partial \Omega, \delta \in[0,1]$.

By the homotopy of degree, we get

$$
\begin{aligned}
\operatorname{deg}(J Q N, \Omega \cap \operatorname{Ker} M, 0) & =\operatorname{deg}(H(\cdot, 0), \Omega \cap \operatorname{Ker} M, 0) \\
& =\operatorname{deg}(H(\cdot, 1), \Omega \cap \operatorname{Ker} M, 0)=\operatorname{deg}(\rho I, \Omega \cap \operatorname{Ker} M, 0) \neq 0 .
\end{aligned}
$$

By Theorem 2.1, we can see that $M u=N u$ has at least one solution in $\bar{\Omega}$. The proof is completed.

Example Let us consider the following boundary value problem at resonance:

$$
\left\{\begin{array}{l}
\left(\varphi_{p}\left(u^{\prime \prime}\right)\right)^{\prime}(t)=\frac{1}{8} t \sin x^{3}+\frac{1}{16} y^{3}+t^{3} \sin z^{3}+\cos t \\
u(0)=u^{\prime \prime}(0)=0, \quad u^{\prime}(1)=2 \int_{0}^{1} t u^{\prime}(t) d t
\end{array}\right.
$$

where $p=4$.

Corresponding to problem (1.1), we have $q=\frac{4}{3}, a(t)=\frac{1}{8} t, b(t)=\frac{1}{16}, c(t)=t^{3}, e(t)=\cos t$, $k(t)=2 t$.

Take $K=4$. By a simple calculation, we find that the conditions $\left(\mathrm{H}_{1}\right)-\left(\mathrm{H}_{4}\right)$ hold. By Theorem 3.1, we obtain the result that problem (3.4) has at least one solution.

\section{The existence of a solution for problem (1.2)}

Let $X=C^{2}[0,1]$ with norm $\|u\|=\max \left\{\|u\|_{\infty},\left\|u^{\prime}\right\|_{\infty},\left\|u^{\prime \prime}\right\|_{\infty}\right\}, Y=C[0,1] \times C[0,1] \times C[0,1]$ with norm $\left\|\left(y_{1}, y_{2}, y_{3}\right)\right\|=\max \left\{\left\|y_{1}\right\|_{\infty},\left\|y_{2}\right\|_{\infty},\left\|y_{3}\right\|_{\infty}\right\}$, where $\|y\|_{\infty}=\max _{t \in[0,1]}|y(t)|$. We know that $(X,\|\cdot\|)$ and $(Y,\|\cdot\|)$ are Banach spaces.

Define operators $M: X \cap \operatorname{dom} M \rightarrow Y, N_{\lambda}: X \rightarrow Y$ as follows:

$$
M u=\left[\begin{array}{c}
\left(\varphi_{p}\left(u^{\prime \prime}\right)\right)^{\prime}(t) \\
T_{1}\left(\varphi_{p}\left(u^{\prime \prime}\right)\right)^{\prime}(t) \\
T_{2}\left(\varphi_{p}\left(u^{\prime \prime}\right)\right)^{\prime}(t)
\end{array}\right], \quad N_{\lambda} u=\left[\begin{array}{c}
\lambda f\left(t, u(t), u^{\prime}(t), u^{\prime \prime}(t)\right) \\
0 \\
0
\end{array}\right]
$$


where $T_{1} y=c_{1}, T_{2} y=c_{2}, y \in C[0,1], c_{1}, c_{2}$ satisfy

$$
\begin{aligned}
& \int_{0}^{1} g(t) \int_{0}^{t} \varphi_{q}\left(\int_{0}^{s} y(r)-c_{1} d r\right) d s d t=0, \\
& \int_{0}^{1} h(t) \int_{t}^{1} \varphi_{q}\left(\int_{0}^{s} y(r)-c_{2} d r\right) d s d t=0, \\
& \operatorname{dom} M=\left\{u \in X \mid \varphi_{p}\left(u^{\prime \prime}\right) \in C^{1}[0,1], u^{\prime \prime}(0)=0\right\} .
\end{aligned}
$$

Lemma 4.1 For $y \in C[0,1]$, there is only one constant $c_{i} \in \mathbb{R}$ such that $T_{i} y=c_{i}$ with $\left|c_{i}\right| \leq$ $\|y\|_{\infty}$. And $T_{i}: C[0,1] \rightarrow \mathbb{R}$ are continuous, $i=1,2$.

The proof is similar to Lemma 3.1.

It is clear that $u \in \operatorname{dom} M$ is a solution if and only if it satisfies $M u=N u$, where $N=N_{1}$. For convenience, let $(a, b, c)^{T}:=\left[\begin{array}{l}a \\ b \\ c\end{array}\right]$.

Lemma 4.2 $M$ is a quasi-linear operator.

Proof It is easy to get $\operatorname{Ker} M=\{a+b t \mid a, b \in \mathbb{R}\}:=X_{1}$.

For $u \in X \cap \operatorname{dom} M$, if $M u=\left(y, c_{1}, c_{2}\right)^{T}$, then $c_{1}, c_{2}$ satisfy (4.1). On the other hand, if $y \in C[0,1], T_{1} y=c_{1}, T_{2} y=c_{2}$, take

$$
u(t)=\int_{0}^{t}(t-s) \varphi_{q}\left(\int_{0}^{s} y(r) d r\right) d s
$$

By simple calculation, we get $u \in X \cap \operatorname{dom} M$ and $M u=\left(y, c_{1}, c_{2}\right)^{T}$. Thus

$$
\operatorname{Im} M=\left\{\left(y, c_{1}, c_{2}\right)^{T} \mid y \in C[0,1], c_{1}, c_{2} \text { satisfy }(4.1)\right\}
$$

By the continuity of $T_{i}, i=1,2$, we see that $\operatorname{Im} M \subset Y$ is closed. So, $M$ is quasi-linear. The proof is completed.

Take a projector $P: X \rightarrow X_{1}$ and an operator $Q: Y \rightarrow Y_{1}$ as follows:

$$
(P u)(t)=u(0)+u^{\prime}(0) t, \quad Q\left(y, y_{1}, y_{2}\right)^{T}=\left(0, T_{1} y_{1}-T_{1} y, T_{2} y_{2}-T_{2} y\right)^{T}
$$

where $Y_{1}=\left\{\left(0, c_{1}, c_{2}\right)^{T} \mid c_{i} \in \mathbb{R}, i=1,2\right\}$. Obviously, $Q Y=Y_{1}$, and $\operatorname{dim} Y_{1}=\operatorname{dim} X_{1}$.

By the continuity and boundedness of $T_{i}, i=1,2$, we can easily see that $Q$ is continuous and bounded in $Y$. It follows from Lemma 3.3 that $Q(I-Q)\left(y, y_{1}, y_{2}\right)^{T}=(0,0,0)^{T}, y, y_{1}, y_{2} \in$ $C[0,1]$.

Define an operator $R: X \times[0,1] \rightarrow X_{2}$ as

$$
R(u, \lambda)(t)=\int_{0}^{t}(t-s) \varphi_{q}\left(\int_{0}^{s} \lambda f\left(r, u(r), u^{\prime}(r), u^{\prime \prime}(r)\right) d r\right) d s
$$

where $\operatorname{Ker} M \oplus X_{2}=X$. By $\left(\mathrm{H}_{2}\right)$ and the Arzela-Asscoli theorem, we can easily see that $R: \bar{\Omega} \times[0,1] \rightarrow X_{2} \cap \operatorname{dom} M$ is continuous and compact, where $\Omega \subset X$ is an open bounded set. 
Lemma 4.3 Assume that $\Omega \subset X$ is an open bounded set. Then $N_{\lambda}$ is $M$-quasi-compact in $\bar{\Omega}$.

Proof It is clear that $\operatorname{Im} P=\operatorname{Ker} M, Q N_{\lambda} x=\theta, \lambda \in(0,1) \Leftrightarrow Q N x=\theta$ and $R(\cdot, 0)=0$. For $u \in \bar{\Omega}$,

$$
\begin{aligned}
(I-Q) N_{\lambda} u & =\left[\begin{array}{c}
\lambda f\left(t, u(t), u^{\prime}(t), u^{\prime \prime}(t)\right) \\
0 \\
0
\end{array}\right]-\left[\begin{array}{c}
0 \\
-T_{1} \lambda f\left(t, u(t), u^{\prime}(t), u^{\prime \prime}(t)\right) \\
-T_{2} \lambda f\left(t, u(t), u^{\prime}(t), u^{\prime \prime}(t)\right)
\end{array}\right] \\
& =\left[\begin{array}{c}
\lambda f\left(t, u(t), u^{\prime}(t), u^{\prime \prime}(t)\right) \\
T_{1} \lambda f\left(t, u(t), u^{\prime}(t), u^{\prime \prime}(t)\right) \\
T_{2} \lambda f\left(t, u(t), u^{\prime}(t), u^{\prime \prime}(t)\right)
\end{array}\right] \in \operatorname{Im} M .
\end{aligned}
$$

Since $\operatorname{Im} M \subset \operatorname{Ker} Q$ and $y=Q y+(I-Q) y$, we obtain $\operatorname{Im} M \subset(I-Q) Y$. Thus, $(I-Q) N_{\lambda}(\bar{\Omega}) \subset$ $\operatorname{Im} M \subset(I-Q) Y$.

For $u \in \Sigma_{\lambda}=\left\{u \in \bar{\Omega} \cap \operatorname{dom} M: M u=N_{\lambda} u\right\}$, we get

$$
\begin{aligned}
R(u, \lambda) & =\int_{0}^{t}(t-s) \varphi_{q}\left(\int_{0}^{s} \lambda f\left(r, u(r), u^{\prime}(r), u^{\prime \prime}(r)\right) d r\right) d s \\
& =\int_{0}^{t}(t-s) \varphi_{q}\left(\int_{0}^{s}\left(\varphi_{p}\left(u^{\prime \prime}\right)\right)^{\prime}\right) d s \\
& =u(t)-u(0)-u^{\prime}(0) t=(I-P) u,
\end{aligned}
$$

i.e. Definition 2.2(c) holds. For $u \in \bar{\Omega}$, we have

$$
M[P u+R(u, \lambda)]=\left[\begin{array}{c}
\lambda f\left(t, u(t), u^{\prime}(t), u^{\prime \prime}(t)\right) \\
T_{1} \lambda f\left(t, u(t), u^{\prime}(t), u^{\prime \prime}(t)\right) \\
T_{2} \lambda f\left(t, u(t), u^{\prime}(t), u^{\prime \prime}(t)\right)
\end{array}\right]=(I-Q) N_{\lambda} u
$$

Thus, Definition 2.2(d) holds. Therefore, $N_{\lambda}$ is $M$-quasi-compact in $\bar{\Omega}$. The proof is completed.

Theorem 4.1 Assume that the following conditions hold:

$\left(\mathrm{H}_{5}\right)$ There exists a nonnegative constant $L$ such that if $|u(t)|>L, t \in[0,1]$ then either

$$
T_{1} f\left(t, u(t), u^{\prime}(t), u^{\prime \prime}(t)\right) \neq 0
$$

or

$$
T_{2} f\left(t, u(t), u^{\prime}(t), u^{\prime \prime}(t)\right) \neq 0 .
$$

$\left(\mathrm{H}_{6}\right)$ There exist nonnegative constants $K_{1}, K_{2}$ such that one of (1) and (2) holds:

(1)

$$
B f(t, A, B, C)>0, \quad t \in[0,1],|B|>K_{1}, A, C \in \mathbb{R},
$$


and

$$
A f(t, A, B, C)>0, \quad t \in[0,1],|B| \leq K_{1},|A|>K_{2}, C \in \mathbb{R} .
$$

(2)

$$
B f(t, A, B, C)<0, \quad t \in[0,1],|B|>K_{1}, A, C \in \mathbb{R},
$$

and

$$
A f(t, A, B, C)<0, \quad t \in[0,1],|A|>K_{2},|B| \leq K_{1}, C \in \mathbb{R} .
$$

$\left(\mathrm{H}_{7}\right)$ There exist nonnegative functions $a(t), b(t), c(t), e(t) \in L^{1}[0,1]$ such that

$$
|f(t, x, y, z)| \leq a(t) \varphi_{p}(|x|)+b(t) \varphi_{p}(|y|)+c(t) \varphi_{p}(|z|)+e(t), \quad t \in[0,1], x, y, z \in \mathbb{R},
$$

where $\varphi_{q}\left(\|a\|_{1}+\|b\|_{1}+\|c\|_{1}\right)<2^{2-q}$, if $1<p \leq 2 ; \varphi_{q}\left(2^{p-2}\|a\|_{1}+2^{p-2}\|b\|_{1}+\|c\|_{1}\right)<1$, if $p \geq 2$.

Then boundary value problem (1.2) has at least one solution.

In order to prove Theorem 4.1, we show two lemmas.

Lemma 4.4 Suppose $\left(\mathrm{H}_{5}\right)-\left(\mathrm{H}_{7}\right)$ hold. Then the set

$$
\Omega_{1}=\left\{u \in \operatorname{dom} M \mid M u=N_{\lambda} u, \lambda \in(0,1)\right\}
$$

is bounded in $X$.

Proof For $u \in \Omega_{1}$, we have $Q N_{\lambda} u=0$, i.e. $T_{i} f\left(t, u(t), u^{\prime}(t), u^{\prime \prime}(t)\right)=0, i=1,2$. By $\left(\mathrm{H}_{5}\right)$ and $\left(\mathrm{H}_{6}\right)$, there exist constants $t_{0}, t_{1} \in[0,1]$ such that $\left|u\left(t_{0}\right)\right| \leq L,\left|u^{\prime}\left(t_{1}\right)\right| \leq K_{1}$. Since $u(t)=$ $u\left(t_{0}\right)+\int_{t_{0}}^{t} u^{\prime}(s) d s, u^{\prime}(t)=u^{\prime}\left(t_{1}\right)+\int_{t_{1}}^{t} u^{\prime \prime}(s) d s$, then

$$
|u(t)| \leq L+\left\|u^{\prime}\right\|_{\infty}, \quad\left|u^{\prime}(t)\right| \leq K_{1}+\left\|u^{\prime \prime}\right\|_{\infty}, \quad t \in[0,1]
$$

It follows from $M u=N_{\lambda} u,\left(\mathrm{H}_{7}\right)$, and (4.2) that

$$
\begin{aligned}
\left|u^{\prime \prime}(t)\right|= & \left|\varphi_{q}\left(\int_{0}^{t} \lambda f\left(s, u(s), u^{\prime}(s)^{\prime} u^{\prime \prime}(s)\right) d s\right)\right| \\
\leq & \varphi_{q}\left(\int_{0}^{1} a(t) \varphi_{p}(|u|)+b(t) \varphi_{p}\left(\left|u^{\prime}\right|\right)+c(t) \varphi_{p}\left(\left|u^{\prime \prime}\right|\right)+e(t) d t\right) \\
\leq & \varphi_{q}\left(\|a\|_{1} \varphi_{p}\left(K_{1}+L+\left\|u^{\prime \prime}\right\|_{\infty}\right)+\|b\|_{1} \varphi_{p}\left(K_{1}+\left\|u^{\prime \prime}\right\|_{\infty}\right)\right. \\
& \left.+\|c\|_{1} \varphi_{p}\left(\left\|u^{\prime \prime}\right\|_{\infty}\right)+\|e\|_{1}\right) .
\end{aligned}
$$

If $1<p \leq 2$, by Lemma 2.1 , we get

$$
\left|u^{\prime \prime}(t)\right| \leq \varphi_{q}\left(B_{1}+A_{1} \varphi_{p}\left(\left\|u^{\prime \prime}\right\|_{\infty}\right)\right) \leq 2^{q-2}\left[\varphi_{q}\left(B_{1}\right)+\varphi_{q}\left(A_{1}\right)\left\|u^{\prime \prime}\right\|_{\infty}\right],
$$


thus

$$
\left\|u^{\prime \prime}\right\|_{\infty} \leq \frac{2^{q-2} \varphi_{q}\left(B_{1}\right)}{1-2^{q-2} \varphi_{q}\left(A_{1}\right)}
$$

where $B_{1}=\|a\|_{1} \varphi_{p}\left(K_{1}+L\right)+\|b\|_{1} \varphi_{p}\left(K_{1}\right)+\|e\|_{1}, A_{1}=\|a\|_{1}+\|b\|_{1}+\|c\|_{1}$.

If $p>2$, by Lemma 2.1, we get

$$
\left|u^{\prime \prime}(t)\right| \leq \varphi_{q}\left(B_{2}+A_{2} \varphi_{p}\left(\left\|u^{\prime \prime}\right\|_{\infty}\right)\right) \leq\left[\varphi_{q}\left(B_{2}\right)+\varphi_{q}\left(A_{2}\right)\left\|u^{\prime \prime}\right\|_{\infty}\right]
$$

thus

$$
\left\|u^{\prime \prime}\right\|_{\infty} \leq \frac{\varphi_{q}\left(B_{2}\right)}{1-\varphi_{q}\left(A_{2}\right)}
$$

where $B_{2}=2^{p-2}\|a\|_{1} \varphi_{p}\left(K_{1}+L\right)+2^{p-2}\|b\|_{1} \varphi_{p}\left(K_{1}\right)+\|e\|_{1}, A_{2}=2^{p-2}\|a\|_{1}+2^{p-2}\|b\|_{1}+\|c\|_{1}$.

These, together with (4.2), mean that $\Omega_{1}$ is bounded in $X$.

Lemma 4.5 Assume $\left(\mathrm{H}_{6}\right)$ holds. Then

$$
\Omega_{2}=\{u \in \operatorname{Ker} M \mid Q N u=0\}
$$

is bounded in $X$, where $N=N_{1}$.

Proof For $u \in \Omega_{2}$, we have $u=a+b t$ and $Q(N u)=0$. By $\left(\mathrm{H}_{6}\right)$, we see that there exists a constant $t_{0} \in[0,1]$ such that $\left|u\left(t_{0}\right)\right|=\left|a+b t_{0}\right| \leq K_{2},\left|u^{\prime}(t)\right|=|b| \leq K_{1}$. So, $\Omega_{2}$ is bounded. The proof is completed.

Proof of Theorem 4.1 Let $\Omega=\{u \in X \mid\|u\|<r\}$, where $r$ is large enough such that $K_{1}+K_{2}<$ $r<+\infty$ and $\Omega \supset \overline{\Omega_{1}} \cup \overline{\Omega_{2}}$.

By Lemmas 4.4 and 4.5, we know $M u \neq N_{\lambda} u, u \in \operatorname{dom} M \cap \partial \Omega$ and $Q N u \neq 0, u \in \operatorname{Ker} M \cap$ $\partial \Omega$.

Let $H(u, \delta)=\rho \delta u+(1-\delta) J Q N u, \delta \in[0,1], u \in \operatorname{Ker} M \cap \bar{\Omega}$, where $J: \operatorname{Im} Q \rightarrow \operatorname{Ker} M$ is a homeomorphism with $J(0, a, b)^{T}=a+b t, \rho= \begin{cases}-1, & \text { if }\left(\mathrm{H}_{6}\right)(1) \text { holds, } \\ 1, & \text { if }\left(\mathrm{H}_{6}\right)(2) \text { holds. }\end{cases}$

Take the function $\operatorname{Sgn}(x)$ is the same as the one in Proof of Theorem 3.1.

For $u \in \operatorname{Ker} M \cap \partial \Omega$, we have $u=a+b t \neq 0$. Thus

$$
H(u, \delta)=\rho \delta(a+b t)+(1-\delta)\left(-T_{1} f(t, a+b t, b, 0)-T_{2} f(t, a+b t, b, 0) t\right) .
$$

If $\delta=1, H(u, 1)=\rho(a+b t) \neq 0$. If $\delta=0$, by $Q N u \neq 0$, we get $H(u, 0)=J Q N(a+b t) \neq 0$. For $0<\delta<1$, we now prove that $H(u, \delta) \neq 0$. Otherwise, if $H(u, \delta)=0$, then

$$
T_{1} f(t, a+b t, b, 0)=\frac{\rho \delta}{1-\delta} a, \quad T_{2} f(t, a+b t, b, 0)=\frac{\rho \delta}{1-\delta} b .
$$

Since $\|u\|=\max \left\{\|a+b t\|_{\infty},|b|\right\}=r>K_{1}+K_{2}$, we have either $|b|>K_{1}$ or $\|a+b t\|_{\infty}>$ $K_{1}+K_{2}$. If $|b|>K_{1}$, then $T_{2} b f(t, a+b t, b, 0)=b T_{2} f(t, a+b t, b, 0)=\frac{\rho \delta}{1-\delta} b^{2}$. So, we have $\operatorname{Sgn}(b f(t, a+b t, b, 0))=\operatorname{Sgn}\left(T_{2} b f(t, a+b t, b, 0)\right)=\operatorname{Sgn}\left(\frac{\rho \delta}{1-\delta} b^{2}\right)=\operatorname{Sgn}(\rho)$. This is a contradiction with the definition of $\rho$. If $|b| \leq K_{1}$, then $\|a+b t\|_{\infty}>K_{1}+K_{2}$. Thus $\min _{t \in[0,1]}|a+b t|>$ 
$K_{2}$ and $\operatorname{Sgn}(a)=\operatorname{Sgn}(a+b t)$. By $T_{1} a f(t, a+b t, b, 0)=a T_{1} f(t, a+b t, b, 0)=\frac{\rho \delta}{1-\delta} a^{2}$, we get $\operatorname{Sgn}\left(T_{1}(a+b t) f(t, a+b t, b, 0)\right)=\operatorname{Sgn}\left(T_{1} a f(t, a+b t, b, 0)\right)=\operatorname{Sgn}\left(\frac{\rho \delta}{1-\delta} a^{2}\right)=\operatorname{Sgn}(\rho)$. This is a contradiction with the definition of $\rho$, too. So, $H(u, \delta) \neq 0, u \in \operatorname{Ker} M \cap \partial \Omega, \delta \in[0,1]$.

By the homotopy of degree, we get

$$
\begin{aligned}
\operatorname{deg}(J Q N, \Omega \cap \operatorname{Ker} M, 0) & =\operatorname{deg}(H(\cdot, 0), \Omega \cap \operatorname{Ker} M, 0) \\
& =\operatorname{deg}(H(\cdot, 1), \Omega \cap \operatorname{Ker} M, 0)=\operatorname{deg}(\rho I, \Omega \cap \operatorname{Ker} M, 0) \neq 0 .
\end{aligned}
$$

By Theorem 2.1, we find that (1.2) has at least one solution in $\bar{\Omega}$. The proof is completed.

\section{Competing interests}

The author declares that she has no competing interests.

\section{Author's contributions}

All results belong to WJ.

\section{Acknowledgements}

This work is supported by the National Science Foundation of China (11171088) and the Natural Science Foundation of Hebei Province (A2013208108).

The author is grateful to anonymous referees for their constructive comments and suggestions, which led to improvement of the original manuscript.

Received: 29 October 2013 Accepted: 24 January 2014 Published: 07 Feb 2014

\section{References}

1. Mawhin, J: Topological degree methods in nonlinear boundary value problems. In: NSFCBMS Regional Conference Series in Mathematics. Am. Math. Soc., Providence (1979)

2. Feng, W, Webb, JRL: Solvability of $m$-point boundary value problems with nonlinear growth. J. Math. Anal. Appl. 212, 467-480 (1997)

3. Liu, Y, Ge, W: Solvability of nonlocal boundary value problems for ordinary differential equations of higher order. Nonlinear Anal. 57, 435-458 (2004)

4. Du, Z, Lin, X, Ge, W: Some higher-order multi-point boundary value problem at resonance. J. Comput. Appl. Math. $177,55-65$ (2005)

5. Liu, B: Solvability of multi-point boundary value problem at resonance (II). Appl. Math. Comput. 136, 353-377 (2003)

6. Zhang, X, Feng, M, Ge, W: Existence result of second-order differential equations with integral boundary conditions at resonance. J. Math. Anal. Appl. 353, 311-319 (2009)

7. Kosmatov, N: Multi-point boundary value problems on an unbounded domain at resonance. Nonlinear Anal. 68 , 2158-2171 (2008)

8. Kosmatov, N: A boundary value problem of fractional order at resonance. Electron. J. Differ. Equ. 2010, 135 (2010)

9. Jiang, W: The existence of solutions to boundary value problems of fractional differential equations at resonance. Nonlinear Anal., Theory Methods Appl. 74, 1987-1994 (2011)

10. Jiang, W: Solvability for a coupled system of fractional differential equations at resonance. Nonlinear Anal.: Real World Appl. 13, 2285-2292 (2012)

11. Del Pino, M, Elgueta, M, Manásevich, R: A homotopic deformation along $p$ of a Leray-Schauder degree result and existence for $\left(\left.\left|u^{\prime}\right|\right|^{p-2} u^{\prime}\right)^{\prime}+f(t, u)=0, u(0)=u(T)=0, p>1$. J. Differ. Equ. 80(1), 3227-3238 (1997)

12. Garcia-Huidobro, M, Manasevich, R, Zanolin, F: A Fredholm-like result for strongly nonlinear second order ODEs. J. Differ. Equ. 114, 132-167 (1994)

13. Garcia-Huidobro, M, Ubilla, P: Multiplicity of solutions for a class of nonlinear second order equations. Nonlinear Anal. 28(9), 1509-1520 (1997)

14. Sun, W, Ge, W: The existence of solutions to Sturm-Liouville BVPs with Laplacian-like operator. Acta Math. Appl. Sin. 18(2), 341-348 (2002)

15. Ge, W, Ren, J: An extension of Mawhin's continuation theorem and its application to boundary value problems with a p-Laplacian. Nonlinear Anal., Theory Methods Appl. 58, 477-488 (2004)

16. Jiang, W: Solvability for $p$-Laplacian boundary value problem at resonance on the half-line. Bound. Value Probl. 2013, 207 (2013). doi:10.1186/1687-2770-2013-207

17. $\mathrm{Du}, \mathrm{B}, \mathrm{Hu}, \mathrm{X}$ : A new continuous theorem for the existence of solutions to $p$-Laplacian BVP at resonance. Appl. Math. Comput. 208, 172-176 (2009) 